\title{
Salmonella Enteritidis Control in Mature Laying Hens Through Dry Fed Parietal Yeast Fraction or Bacillus Blend Probiotic
}

\author{
John Connor Padgett ${ }^{1,2, *}$, Paul Thomas Price ${ }^{2,3}$, James Allen Byrd ${ }^{4}$, Christopher Anthony Bailey ${ }^{1}$ \\ ${ }^{1}$ Department of Poultry Science, Texas A\&M University, College Station, United States \\ ${ }^{2}$ Phileo by Lesaffre, Milwaukee, United States \\ ${ }^{3}$ Department of Food, Nutrition, and Packaging Sciences, Clemson University, Clemson, United States \\ ${ }^{4}$ Agricultural Research Service, United States Department of Agriculture, College Station, United States
}

Email address:

c.padgett@phileo.lesaffre.com (J. C. Padgett), p.price@phileo.lesaffre.com (P. T. Price), allen.byrd2@usda.gov (J. A. Byrd), c-bailey@tamu.edu (C. A. Bailey)

*Corresponding author

\section{To cite this article:}

John Connor Padgett, Paul Thomas Price, James Allen Byrd, Christopher Anthony Bailey. Salmonella Enteritidis Control in Mature Laying Hens Through Dry Fed Parietal Yeast Fraction or Bacillus Blend Probiotic. International Journal of Animal Science and Technology. Vol. 5, No. 1, 2021, pp. 1-6. doi: 10.11648/j.jjast.20210501.11

Received: January 7, 2021; Accepted: January 19, 2021; Published: January 25, 2021

\begin{abstract}
With food safety continuing to be a concern for both consumers and producers, especially when considering Salmonella, alternative control methods must be studied intensively to fully understand their effects in live poultry. To further understand the abilities of parietal yeast fractions and bacillus probiotics to each impact food safety, a study on mature laying hens was undertaken. 48 hens were obtained from a commercial production flock at 75 weeks of age and were transferred to the USDA ARS facility in College Station, TX. The hens were randomly divided into 3 treatment groups, and fed either a mash corn soy control diet (CON), a corn soy mash diet plus parietal yeast fration composed of a minimum of $20 \%$ mannan and $20 \%$ beta 1,3 1,6 glucans (YF), or a corn soy mash diet plus a three-strain bacillus probiotic (3B). After 3 weeks of receiving dietary treatment, all birds were orally challenged with $6.6 \times 10^{9} \mathrm{CFU}$ of Salmonella Enteritidis. One week after being challenged with Salmonella Enteritidis, birds were humanely euthanized, and ovary and cecal contents were removed and direct plated for both prevalence and enumeration. After plating cecal contents, a significant reduction of $1.26 \log 10$ was observed from the CON to YF $(p=0.03)$ and a $1.08 \log 10$ reduction was observed from $C O N$ to $3 B(p=0.04)$. Prevalence of ovaries was not significantly different, but numerically CON samples were $50 \%$ positive, while YF and $3 \mathrm{~B}$ were $25 \%$ and $13 \%$ positive respectively. Prevalence in the ceca was not significantly different, but numerically CON samples were $75 \%$ positive, while YF and $3 \mathrm{~B}$ were $44 \%$ and $53 \%$ respectively. While neither ovary or ceca prevalence were significantly reduced in this experiment with the inclusion of either treatment, both the YF and 3B treatment significantly reduced colonization of Salmonella Enteritidis in the ceca, indicating that both treatments possess excellent potential as food safety interventions against S. Enteritidis in the egg supply chain.
\end{abstract}

Keywords: Salmonella, Enteritidis, Layers, Laying Hens, Yeast Cell Wall, Yeast Fraction, Bacillus, Probiotic

\section{Introduction}

Salmonellosis is one of the leading causes of food poisoning in the United States and is responsible for over 26,500 hospitalizations \& over 400 deaths annually [1]. The illness is caused by Salmonella spp. and usually is accompanied by fever, diarrhea, and stomach cramps. While most people recover within 4-7 days, those who are particularly at risk for more severe illness are people with compromised immune systems, elderly patients (over 65 years), or infants under 12 months. Infection usually occurs from consumption of food or water contaminated by animals and their feces [2]. According to Foodnet in 2019 Salmonella was the second leading cause of 
foodborne illness at $17 \%$ behind only Campylobacter [3]. Salmonella is a gram negative facultative anaerobe, of which there are over 2500 known serovars and around 100 that cause disease in humans [4]. The bacteria are flagellated, containing $\mathrm{O}, \mathrm{H}$, or $\mathrm{Vi}$ antigens which can be detected by the immune system. Their ability to pass through the acidic environment of the GI tract allows them to invade both mucosa and epithelial cells. Their uptake to macrophages \& direct invasion of epithelial cells can trigger inflammation through the release of proinflammatory cytokines [5]. As most Salmonella is ingested orally, the defenses in place to mitigate colonization can include the peristalsis motility of the intestine, the gastric acid level of the stomach, and the competing microflora of the intestine. It is when an environment such as the microflora becomes disrupted that infection occurs through proliferation and increased colonization $[6,7]$.

Since 2007, S. Enteritidis has been the most commonly isolated serovar from reported illnesses without decreasing [1]. Salmonellosis cases where this organism is identified have been associated with consumption of poultry products, and significant associations have been made between this serovar to eggs and egg containing foods [8]. Due to the virulence genes it possesses, S. Enteritidis can survive the intestinal tract, multiply within the intestine through the macrophages, liver, and migrate to colonize the ovaries \& preovulatory follicles of hens $[9,10]$. To combat the prevalence of this organism in the food supply, the FDA issued the egg safety rule mandating pullet sourcing protocols, disinfection, environmental monitoring, and fly/rodent control. FDA also advises producers to utilize biosecurity on the farm, as well as vaccination protocols involving attenuated live strains $\&$ killed bacterins. It has been suggested that utilization of feed \& water additives known to impact the population of S. Enteritidis in the hen \& environment is part of a successful plan [11].

A number of feed additives have shown efficacy in the GI tract of production poultry against S. Enteritidis, such as butyrate, yeast \& probiotics which have established repeated successes [12-15]. Yeast components included as fractions and fermentations often contain high levels of mannans and beta 1-3, 1-6 glucans. These particular prebiotic components have shown to be particularly effective against some Salmonella Enteritidis colonization in chicks, broilers, pullets, and mature laying hens [16-20]. Yeast and bacillus probiotics are often utilized in the commercial poultry industry for other health \& production benefits, and many different bacillus strains have shown effective impact on S. Enteritidis [21-23]. These organisms which sporulate in a protective state are often sought for their stability in the volatile poultry feed delivery system. They have been known to secrete enzymes that can aid in the digestion of non starch polysaccharides, \& hydrolyze bacterial toxins, possibly improving the microflora profile of the gut [24, 25]. In addition to their efficacy against intestinal challenges such as coccidiosis induced necrotic enteritis, bacillus probiotics have been used for their antimicrobial compound secretion and suppression of S. Enteritidis growth [26, 27]. Due to previously published efficacy in pullets, a commercially available premium yeast fraction (SafMannan) and a commercially available bacillus probiotic blend (MicroSaf) were utilized in this study to evaluate ability to inhibit S. Enteritidis in mature laying hens [28, 29].

\section{Materials and Methods}

\subsection{Bird Husbandry and Management}

All procedures of this study were approved and are in accordance with local Institutional Animal Care and Use Committee (IACUC) guidelines. A total of 48 Lohmann brown laying hens were obtained from a commercial facility and transported to the USDA - ARS facilities in College Station, TX. A basal laying hen diet was formulated to meet or exceed birds needs according to nutritional recommendations for Lohmann brown laying hens (Table 1). The basal diet was divided into 3 batches to serve as treatment groups. The first batch remained as a basal diet to serve as the control group (CON). The second batch was supplemented with a parietal yeast fraction (YF) composed of a minimum $20 \%$ mannan and $20 \%$ beta 1,3 1,6 glucans at 500ppm. The third batch was supplemented with a three-strain bacillus probiotic (3B) composed of Bacillus amyloliquefaciens, Bacillus licheniformis, and Bacillus pumilus at 500ppm.

Table 1. Laying Hen Diet.

\begin{tabular}{ll}
\hline Ingredients & Percentage \\
\hline Corn & 63.62 \\
Soybean meal & 20.59 \\
DL-Methionine & 0.17 \\
Soybean Oil & 1.86 \\
Limestone & 11.66 \\
Biofos & 1.33 \\
Salt & 0.24 \\
Sodium Bicarbonate & 0.23 \\
Trace Minerals ${ }^{1}$ & 0.05 \\
Trace Vitamins & \\
\hline
\end{tabular}

${ }^{1}$ Per pound of premix; $\mathrm{Cu}$ : Copper minimum $1.40 \%$, I: Iodine minimum 800.0ppm, FE: Iron minimum 12.00\%, Mm: Manganese minimum 12.00\%, $\mathrm{Zn}$ : Zinc minimum $12.00 \%$, ${ }^{2}$ Per pound of premix; Vitamin A: 4,000,000 IU, Vitamin D3: 1,400,000 IU, Vitamin E: 16,666 IU, Vitamin B12: 6mg, Riboflavin (B2): 2166mg, Niacin (B3): 16,666mg, d-pantothenic acid (B5): 7334mg, Choline: 47383mg, Menadione: 534mg, Folic acid (B9): 634mg, Pyridoxine (B6): 2,600mg, Thiamine (B1): 1,066mg, d-Biotin (B7): 200mg.

Birds were randomly divided into control (CON) or treatment (YF or 3B) treatment groups (16 birds in each group) and allocated into stainless steel A-frame laying hen cages (2 birds per cage). Birds were housed in an environmentally controlled rearing room equipped with feeders and nipple drinkers. Birds were observed daily regarding temperature, feed, water, egg production, and general flock condition. Egg production factors were recorded at the end of each day.

\subsection{Data Collection: Prevalence and Enumeration}

Prior to challenging the birds with Salmonella Enteritidis, all hens were tested for Salmonella prevalence via a cloacal swab. Swabs were placed into conical tubes containing RV broth, homogenized by shaking, and incubated for 24 hours. After 24 
hours of incubation a $10 \mu \mathrm{L}$ sterile loop was used to streak plate a sample onto Xylose-Lysine-Tergitol 4 (XLT-4) Agar (Hardy Diagnostics, Santa Maria, CA). Samples were deemed positive after 24 hours of incubation. Any bird found to be positive prior to the challenge was removed from the study.

After a 3-week acclimation period, birds were challenged with $2.2 \times 10^{9} \mathrm{CFU} / \mathrm{mL}$ of Salmonella Entertitidis by oral gavage ( $3 \mathrm{~mL}$ gavage per bird). Three days after challenging with Salmonella Enteritidis, a cloacal swab was taken from each bird and direct plated onto XLT-4 agar treated with Novobiocin $(20 \mathrm{mg} / \mathrm{mL})$ and Nalidixic acid $(25 \mathrm{mg} / \mathrm{mL})$ to ensure that the challenge was successful. The challenge was deemed successful if at least $25 \%$ positive prevalence was found through direct plating of cloacal swabs 3 days post challenge. One week after the initial Salmonella challenge, the hens were humanely euthanized, and samples of the ceca and ovary were taken for further analysis.

Ceca samples taken at termination were divided in half. One cecum was placed into conical tube containing Rappaport Vassiliadis (RV) (Difco, Franklin Lakes, NJ) broth for use as an enrichment method in prevalence determination. For ovary samples, a small sample of ovaries were obtained and placed into a conical tube containing RV broth. Samples were homogenized by shaking and incubated for 24 hours. After 24 hours of incubation, samples were again homogenized by shaking and a $10 \mu \mathrm{L}$ sterile loop was used to plate a sample onto XLT-4 agar. Samples were identified as positive by visual identification of colonies on the plates after 24 hours of incubation.

For ceca counts, the other cecum was diluted using a $10 \mathrm{x}$ dilution series and plated onto XLT-4 Agar treated with Novobiocin $(20 \mathrm{mg} / \mathrm{mL})$ and Nalidixic acid $(25 \mathrm{mg} / \mathrm{mL})$ for use as a selective growth media. Counts were determined by visual inspection of colonies after 48 hours of incubation.

\subsection{Data Analysis}

Data obtained during this trial were analyzed using a one way analysis of variance (ANOVA) and means separated using students t-test were considered statistically significantly difference at $p \leq 0.05$. The ANOVA was used to compare observations between treatment groups for each of the factors (ceca and ovary prevalence, ceca enumeration) observed during this study as noted in the data collection section. Mean and standard deviation values were used in the determination of outliers as well as for comparisons between treatment groups. All data were analyzed using STATA v. 16.1 (StataCorp, College Station, TX).

\section{Results}

For cecal content analysis, Control Salmonella results prevalence (Figure 1) showed 12 of 16 birds (75\%) positive. Safmannan treatment showed 7 of 16 birds positive (44\%), and MicroSaf showed 8 of 15 birds positive (53\%).

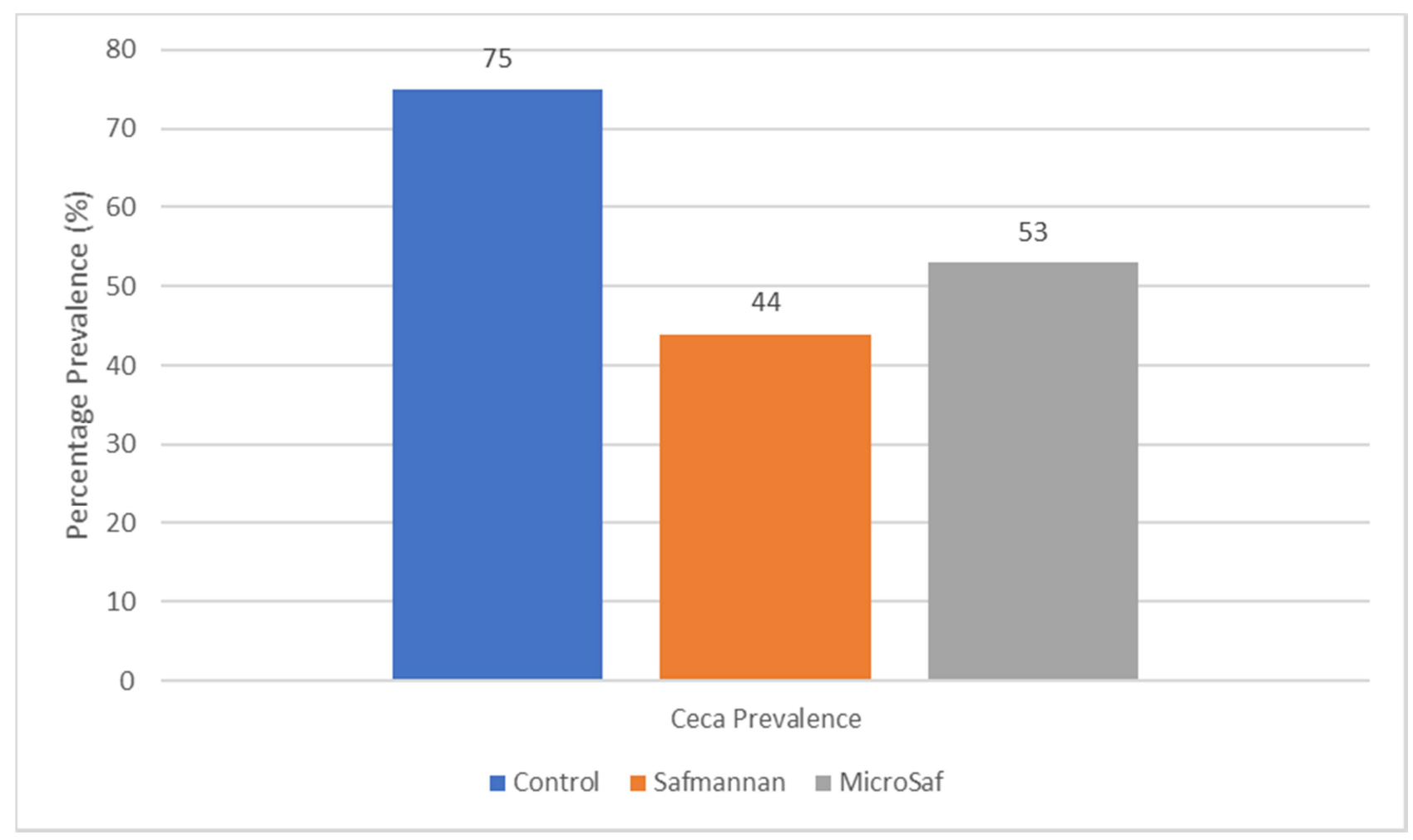

Figure 1. Ceca Percentage Prevalence.

The results in Figure 2 show Ovary prevalence following the trend observed of treatments reducing prevalence as they did in ceca, with control birds 8 of 16 positive $(50 \%)$,
Safmannan birds 4 of 16 positive (25\%), and MicroSaf birds 2 of 15 positive $(13 \%)$. None of these results were significant at the $\mathrm{p}=.05$ level. 


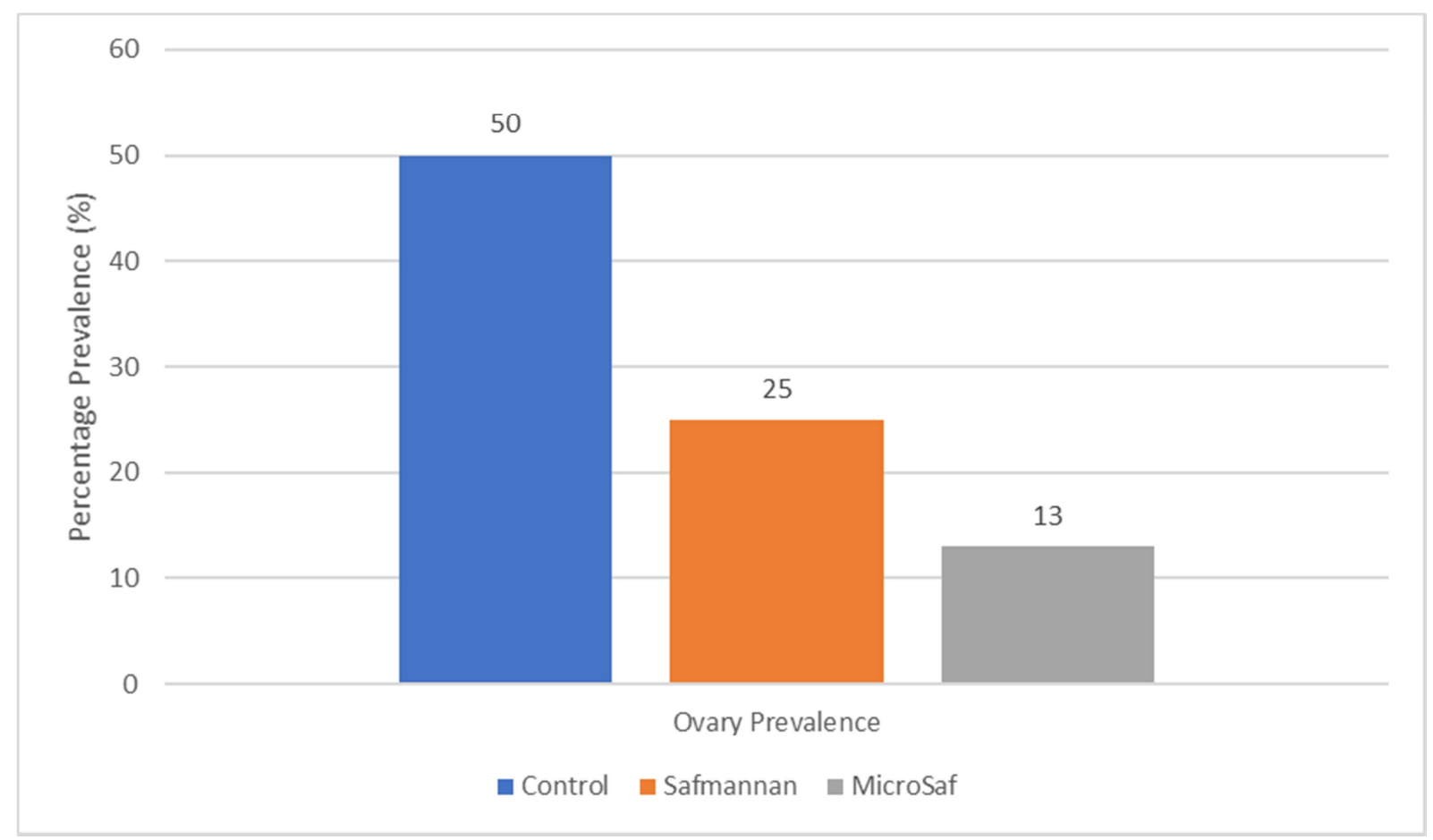

Figure 2. Ovary Percentage Prevalence.

However, results observed for enumeration of cecal culture showed significant reduction of S. E. for Saf Mannan (762.5 $\mathrm{CFU} / \mathrm{g}, \mathrm{p}=.0374)$ and MicroSaf (1156.7 CFU/g, p=.0466) when each was compared to Control (13762.5CFU/g) (Table
2). When log converted the difference was over a log between SafMannan \& Control (Log1.26) and MicroSaf \& Control (Log1.08) (Table 2, Figure 3).

Table 2. CFU/g and Log10 CFU/g of Ceca Content.

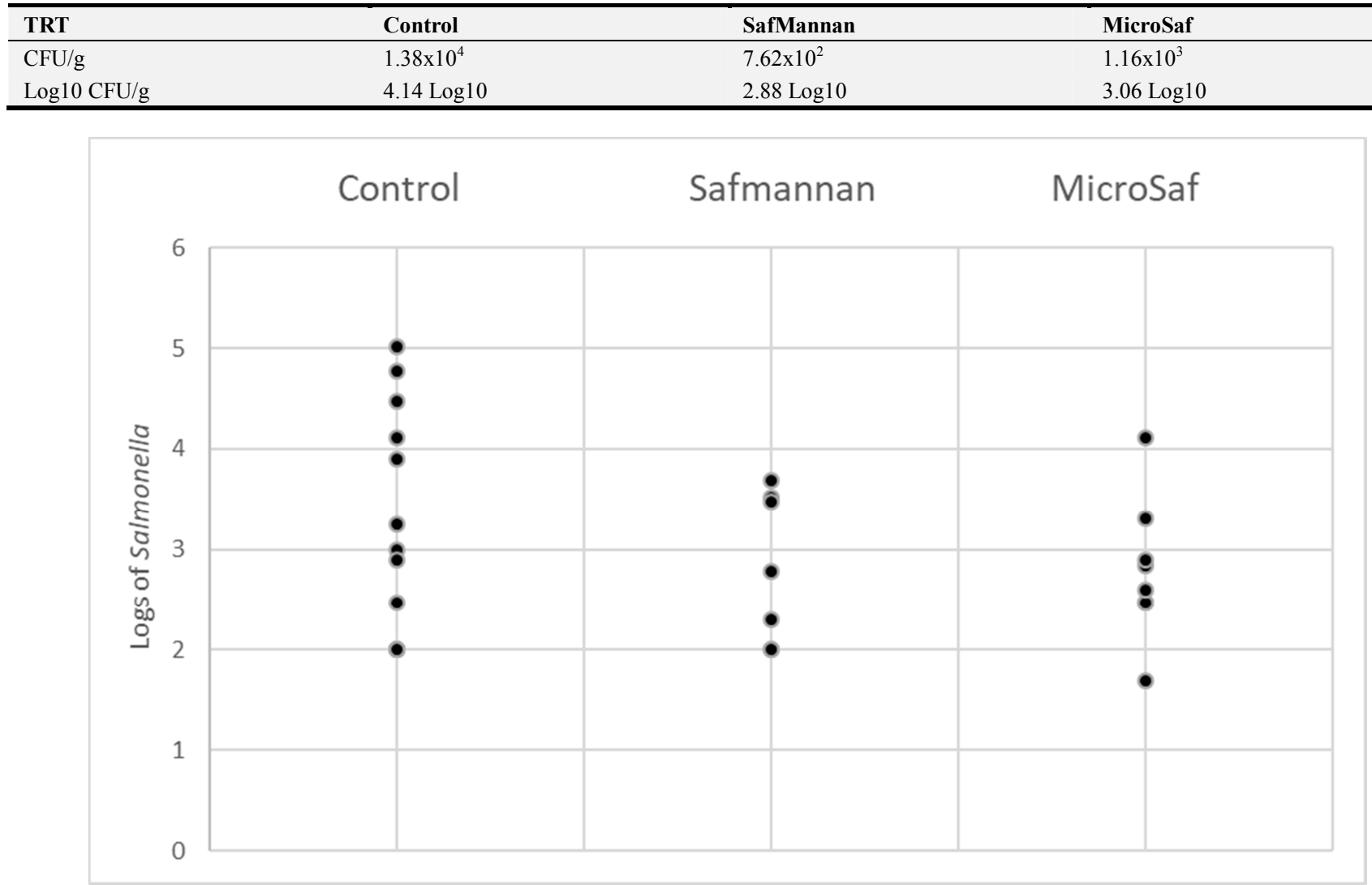

Figure 3. Log10 CFU/g of Ceca Content. 


\section{Discussion}

Yeast fractions and yeast cell wall including SafMannan have also shown binding affinity with a wide range of pathogens, including varying binding affinity with a range of Salmonella serotypes. This binding affinity promotes adhesion through direct agglutination of the pathogenic microbes to the mannose in yeast fractions or yeast cell wall via the type-1 fimbriae, thus resulting in the ultimate removal of the pathogen from the gastrointestinal tract (GIT) [32, 33]. Bacillus products have been shown as well to have promising activity in the intestinal tract through competitive exclusion, as well as the facilitation of cross-talk between the gut microbes and the immune system, resulting in decreased gut inflammation [34-36].

It has been observed that most $\mathrm{S}$. Enteritidis contaminated eggs contain less than $10 \mathrm{CFU}[30,31]$. With such a low level of infection in flocks a reduction in the GI tract of $1 \log$ would indicate a high level of biological significance in impacting $\mathrm{S}$. Enteritidis colonization. As yeast \& bacillus probiotics are known to reduce colonization sites in the microvilli of the small intestine, and decrease leaky gut conditions that allow Salmonella to enter the macrophages or blood stream \& eventually reproductive tract, it would make sense that a reduction in cecal population of S. Enteritidis. should lead to lower positive samples in the ovary [37-39].

SafMannan premium yeast fraction has previously shown reductions of S. Enteritidis prevalence in the ceca, as well as reduction against $\mathrm{S}$. Typhimurium by a $\log [28,29]$. MicroSaf has been observed to reduce S. Enteritidis in layer ceca to levels approaching a $\log [28]$.

\section{Conclusion}

While reductions in cecal and ovary prevalence were not statistically significant, both SafMannan (YF) and MicroSaf (3B) showed reductions in prevalence in both categories. Both SafMannan and MicroSaf showed statistically significant reductions in cecal $\mathrm{CFU} / \mathrm{g}$, indicating that both SafMannan and MicroSaf have excellent potential as food safety interventions against $\mathrm{S}$. Enteritidis in the egg supply chain.

\section{Conflict of Interest}

The authors declare that they have no competing interests.

\section{Acknowledgements}

This work was funded by Phileo by Lesaffre through Project \#66950 under the direction of Dr. Christopher A. Bailey at Texas A\&M University.

\section{References}

[1] CDC https://www.cdc.gov/salmonella/.

[2] European Food Safety Authority (EFSA), European Centre for
Disease Prevention and Control (ECDC). The European Union summary report on trends and sources of zoonoses, zoonotic zgents and food-borne outbreaks in 2012. EFSA Journal. 2014; 12: 3547 .

[3] Foodnet https://www.cdc.gov/mmwr/volumes/69/wr/mm6917al.htm?s _cid=mm6917al_w.

[4] Underwood, W. J., R. Blauwiekel, M. L. Delano, R. Gillesby, S. A. Mischler, A. Schoell. Chapter 15 - Biology and Diseases of Ruminants (Sheep, Goats, and Cattle). Editor (s): Fox, JG Anderson, LC, Otto, GM, Pritchett-Corning, KR. Whary, M. T. In American College of Laboratory Animal Medicine, Laboratory Animal Medicine (Third Edition), Academic Press, 2015. 623-694.

[5] Giannella RA. Importance of the intestinal inflammatory reaction in Salmonella-mediated intestinal secretion. Infect Immune. 1979; 23: 140.

[6] Giannella RA, Formal SB, Dammin GJ. et al. Pathogenesis of salmonellosis. Studies of fluid secretion, mucosal invasion, and morphologic reaction in the rabbit ileum. J Clin Invest. 1973; 52: 441 .

[7] Finlay BB, Leung KY, Rosenshine I. et al. Salmonella interactions with the epithelial cell. A model to study the biology of intracellular parasitism. ASM News. 1992; 58: 486.

[8] Gast RK, Guard-Bouldin J, Holt PS. Colonization of reproductive organs and internal contamination of eggs after experimental infection of laying hens with Salmonella heidelberg and Salmonella enteritidis. Avian Dis. 2004 Dec; 48 (4): 863-9.

[9] Foley SL, Nayak R, Hanning IB, Johnson TJ, Han J, Ricke SC. Population dynamics of Salmonella enterica serotypes in commercial egg and poultry production. Appl Environ Microbiol. 2011; 77 (13): 4273-4279.

[10] Velge P, Cloeckaert A, Barrow P. Emergence of Salmonella epidemics: the problems related to Salmonella enterica serotype Enteritidis and multiple antibiotic resistance in other major serotypes. Vet Res. 2005 May-Jun; 36 (3): 267-88.

[11] Food and Drug Administration 2010. Guidance for industry: prevention of Salmonella Enteritidis in shell eggs during production, transportation, and storage; small entity compliance guide. U.S. Department of Health and Human Services, FDA, Rockville, MD.

[12] Fernández-Rubio C, Ordóñez C, Abad-González J, Garcia-Gallego A, Honrubia MP, Mallo JJ, Balaña-Fouce R. Butyric acid-based feed additives help protect broiler chickens from Salmonella Enteritidis infection. Poult Sci. 2009 May; 88 (5): 943-8.

[13] Van Immerseel, F., Cauwerts, K., Devriese, L., Haesebrouck, F., \& Ducatelle, R. (2002). Feed additives to control Salmonella in poultry. World's Poultry Science Journal, 58 (4), 501-513.

[14] Bailey, J. S., Blankenship, L. C. and Cox, N. A. (1991) Effect of fructooligosaccharide on Salmonella colonization of the chicken intestine. Poultry Science 70: 2433-2438.

[15] Collins, M. D. and Gibson, R. (1999) Probiotics, prebiotics and synbiotics: approaches for modulating the microbial ecology of the gut. American Journal of Clinical Nutrition 69: 1052S$1057 \mathrm{~S}$. 
[16] Girgis G, Powell M, Youssef M, Graugnard DE, King WD, et al. (2020) Effects of a mannan-rich yeast cell wall-derived preparation on cecal concentrations and tissue prevalence of Salmonella Enteritidis in layer chickens. PLOS ONE 15 (4): e0232088.

[17] Charles L. Hofacre, Roy D. Berghaus, Sangita Jalukar, Greg F. Mathis, John A. Smith. Effect of a Yeast Cell Wall Preparation on Cecal and Ovarian Colonization With Salmonella enteritidis in Commercial Layers. 2018. Jour Applied Poul Research 27: (4), 453-460, ISSN 1056-6171.

[18] Attia, Y. A., Ellakany, H. F., Abd El-Hamid, A. E., Bovera, F., Ghazaly, S. A. Control of Salmonella enteritidis infection in male layer chickens by acetic acid and/or prebiotics, probiotics and antibiotics. 2012. Arch. Geflügelk., 76 (4). S. 239-245.

[19] Price P. T, Gaydos T, Padgett J. C, Gardner K, Bailey C. Salmonella colonization of production hens fed a parietal yeast fraction with high levels of mannan and beta-glucan. Int. J. Poult. Sci. 2019; 18 (9): 410-415.

[20] Lourenço M. C, Kuritza L. N, Hayashi R. M, Miglino L. B, Durau J. F, Pickler L, Santin E. Effect of a mannanoligosaccharide-supplemented diet on intestinal mucosa $\mathrm{T}$ lymphocyte populations in chickens challenged with Salmonella Enteritidis. J. Appl. Poult. Res. 2015; 24 (1): 1522.

[21] Wenrui Zhen, Yujing Shao, Xiuyan Gong, Yuanyuan Wu, Yanqiang Geng, Zhong Wang, Yuming Guo. Effect of dietary Bacillus coagulans supplementation on growth performance and immune responses of broiler chickens challenged by Salmonella enteritidis. Poult Sci. 2018.97 (8): 2654-2666.

[22] Murate, Letícia \& Paião, Fernanda \& Almeida, Adriana \& Jr, Angelo \& Shimokomaki, Massami. (2015). Efficacy of Prebiotics, Probiotics, and Synbiotics on Laying Hens and Broilers Challenged with Salmonella Enteritidis. The Journal of Poultry Science. 52. 52-56.

[23] Adhikari B, Hernandez-Patlan D, Solis-Cruz B, et al. Evaluation of the Antimicrobial and Anti-inflammatory Properties of Bacillus-DFM (Norum ${ }^{\mathrm{TM}}$ ) in Broiler Chickens Infected With Salmonella Enteritidis. Front Vet Sci. 2019; 6: 282. Published 2019 Aug 27.

[24] Khan, S., Chousalkar, K. K. Salmonella Typhimurium infection disrupts but continuous feeding of Bacillus based probiotic restores gut microbiota in infected hens. 2020. J Animal Sci Biotechnol 11, 29.

[25] R. Shanmugasundaram, T. J. Applegate, R. K. Selvaraj. Effect of Bacillus subtilis and Bacillus licheniformis probiotic supplementation on cecal Salmonella load in broilers challenged with salmonella. 2020. Journal of Applied Poultry Research. 29 (4): 808-816.

[26] A. Knarreborg, E. Brockmann, K. Høybye, I. Knap, B. Lund, N. Milora, T. D. Leser. Bacillus subtilis (DSM17299) modulates the ileal microbial communities and improves growth performance in broilers. 2008. Int. J. Probiotics Prebiotics, (3) pp. 83-88.

[27] I. Knap, A. B. Kehlet, M. Bennedsen, G. F. Mathis, C. L.
Hofacre, B. S. Lumpkins, M. M. Jensen, M. Raun, A. Lay. Bacillus subtilis (DSM17299) significantly reduces Salmonella in broilers. 2011. Poult. Sci., (90) 1690-1694.

[28] Price PT, Gaydos TA, Berghaus RD, Baxter V, Hofacre CL, Sims MD. Salmonella Enteritidis reduction in layer ceca with a Bacillus probiotic. Vet World. 2020; 13 (1): 184-187. doi: 10.14202/vetworld.2020.184-187.

[29] Price, PT, Gaydos, TA, Berghaus, RD, Hofacre, CL. Reduction of Salmonella Enteritidis Colonization in Production Layers Fed High Levels of Mannan and Beta-glucan. 2020. Asi. Jour. Poult. Sci. 14 (1): 1-5.

[30] Humphrey T. J., Naskerville A., Mawer S., Rowe B., Hopper S. Salmonella Enteritidis phage type 4 from the contents of intact eggs: A study involving naturally infected hens. Epidemiol. Infect., 104 (1989), pp. 415-423.

[31] Gast R. K., Beard C. W. Production of Salmonella Enteritidis-contaminated eggs by experimentally infected hens. Avian Dis., 34 (1990), pp. 438-446.

[32] Posadas, G. A., Broadway, P. R., Thornton, J. A., Carroll, J. A., Lawrence, A., Corley, J. R., Thompson, A., \& Donaldson, J. R. (2017). Yeast Pro- and Paraprobiotics Have the Capability to Bind Pathogenic Bacteria Associated with Animal Disease 1. Translational Animal Science, 1 (1), 60-68.

[33] Akhtar T, Ara G, Ali N, Ud Din Mufti F, Imran Khan M. Effects of dietary supplementation of mannanoligosaccharide on virus shedding in avian influenza (H9N2) challenged broilers. Iran J Vet Res. 2016; 17 (4): 268-272.

[34] Kabir, S. M. L. (2009). The Role of Probiotics in the Poultry Industry. International Journal of Molecular Sciences, 10 (8), 3531-3546.

[35] Al-Khalaifa, H., Al-Nasser, A., Al-Surayee, T., Al-Kandari, S., Al-Enzi, N., Al-Sharrah, T., Ragheb, G., Al-Qalaf, S., \& Mohammed, A. Effect of dietary probiotics and prebiotics on the performance of broiler chickens. 2019. Poultry Science, 98 (10), 4465-4479.

[36] Al-Khalaifah, H. S. Benefits of probiotics and/or prebiotics for antibiotic-reduced poultry. Poultry Science. 2018. 97 (11), 3807-3815.

[37] Wang W, Li Z, Han Q, Guo Y, Zhang B, D'inca R. Dietary live yeast and mannan-oligosaccharide supplementation attenuate intestinal inflammation and barrier dysfunction induced by Escherichia coli in broilers. Br J Nutr. 2016 Dec; 116 (11): 1878-1888.

[38] Nopvichai C, Charoenwongpaiboon T, Luengluepunya N, Ito K, Muanprasat C, Pichyangkura R. Production and purification of mannan oligosaccharide with epithelial tight junction enhancing activity. PeerJ. 2019; 7: e7206. Published 2019 Jul 2.

[39] Gharib-Naseri, K., de Paula Dorigam, J. C., Doranalli, K. et al. Modulations of genes related to gut integrity, apoptosis, and immunity underlie the beneficial effects of Bacillus amyloliquefaciens CECT 5940 in broilers fed diets with different protein levels in a necrotic enteritis challenge model. J Animal Sci Biotechnol 11, 104 (2020). 